\title{
A GENERAL FORMALISM FOR SINGLE AND DOUBLE SPIN ASYMMETRIES IN INCLUSIVE HADRON PRODUCTION *
}

\author{
U. D'ALESIO ${ }^{\dagger}$ S. MELIS \\ Istituto Nazionale di Fisica Nucleare, Sezione di Cagliari \\ and Dipartimento di Fisica, Università di Cagliari \\ C.P. 170,I-09042 Monserrato (CA), Italy
}

\begin{abstract}
We present a calculation of single and double spin asymmetries for inclusive hadron production in hadronic collisions. Our approach is based on Leading Order (LO) perturbative QCD and generalized factorization theorems, with full account of intrinsic parton momentum, $\boldsymbol{k}_{\perp}$, effects. This leads to a new class of spin and $\boldsymbol{k}_{\perp}-$ dependent distribution and fragmentation functions. Limiting ourselves to consider leading twist functions, we show how they could play a relevant role in producing non-vanishing spin asymmetries.
\end{abstract}

\section{Introduction}

It has been experimentally known since a long time that transverse single spin asymmetries (SSA) in hadronic collisions can be, in particular kinematical regions, very large. Two relevant examples are: the transverse $\Lambda$ polarization, $P_{T}^{\Lambda}$, measured in unpolarized hadron collisions; the SSA, $A_{N}$, observed in $p^{\uparrow} p \rightarrow \pi+X . P_{T}^{\Lambda}$ and $A_{N}$ are defined as:

$$
P_{T}^{\Lambda}=\frac{\mathrm{d} \sigma^{A B \rightarrow \Lambda^{\uparrow} X}-\mathrm{d} \sigma^{A B \rightarrow \Lambda^{\downarrow} X}}{\mathrm{~d} \sigma^{A B \rightarrow \Lambda^{\uparrow} X}+\mathrm{d} \sigma^{A B \rightarrow \Lambda^{\downarrow} X}} \quad A_{N}=\frac{\mathrm{d} \sigma^{A^{\uparrow} B \rightarrow C X}-\mathrm{d} \sigma^{A^{\downarrow} B \rightarrow C X}}{\mathrm{~d} \sigma^{A^{\uparrow} B \rightarrow C X}+\mathrm{d} \sigma^{A^{\downarrow} B \rightarrow C X}}
$$

where $\mathrm{d} \sigma$ stands for the corresponding invariant differential cross section and $\uparrow, \downarrow$ denote transverse polarization with respect to the hadron production plane. Both these observables can reach in size values up to $30 \%$ $40 \%$. These results are at first puzzling in the context of perturbative QCD

*Talk delivered by S. Melis at the "X Convegno su Problemi di Fisica Nucleare Teorica", October 6-9, 2004, Cortona, Italy.

${ }^{\dagger}$ E-mail: umberto.dalesio@ca.infn.it

‡E-mail: stefano.melis@ca.infn.it

$\S$ E-mail: francesco.murgia@ca.infn.it 
(pQCD) if, as usual, one assumes a collinear partonic kinematics. In fact, it is easy to see that in this case pQCD predicts almost vanishing SSA at large energy scales. Writing a transverse spin state as a combination of helicity states, $|\uparrow / \downarrow\rangle=\frac{1}{\sqrt{2}}[|+\rangle \pm i|-\rangle]$, we have schematically:

$$
A_{N} \sim \frac{\langle\uparrow \mid \uparrow\rangle-\langle\downarrow \mid \downarrow\rangle}{\langle\uparrow \mid \uparrow\rangle+\langle\downarrow \mid \downarrow\rangle} \sim \frac{2 \operatorname{Im}\langle+\mid-\rangle}{\langle+\mid+\rangle+\langle-\mid-\rangle},
$$

where $\langle\uparrow \mid \uparrow\rangle$ stands for the partonic cross section $\mathrm{d} \hat{\sigma}^{\uparrow}$. The presence of a term like $\operatorname{Im}\langle+\mid-\rangle$ in the numerator of Eq. (2) requires both helicity flip at the partonic level and a relative phase between helicity amplitudes. Because massless QCD conserves helicity and Born level amplitudes are real, $A_{N}$ should be proportional to the quark mass times a factor $\alpha_{s}$ coming from the required higher order contributions. This implies $A_{N} \propto \alpha_{s} m_{q} / E_{q}$, which would be negligible at large energies. A possible way out is to take into account partonic transverse momenta, $\boldsymbol{k}_{\perp}$, in parton distribution and fragmentation functions and in the elementary scattering process. This in turn leads to the introduction of a new class of spin and $\boldsymbol{k}_{\perp}$ dependent partonic distribution (PDF) and fragmentation (FF) functions that, at least in principle, are able to generate spin asymmetries.

\section{General formalism}

In the usual collinear pQCD approach at leading order (LO) and leading twist, the unpolarized cross section for the process $A B \rightarrow C X$ reads:

$$
\begin{aligned}
\mathrm{d} \sigma^{A B \rightarrow C X}= & \sum_{a, b, c, d} f_{a / A}\left(x_{a}, Q^{2}\right) \otimes f_{b / B}\left(x_{b}, Q^{2}\right) \\
& \otimes \mathrm{d} \hat{\sigma}^{a b \rightarrow c d}\left(\hat{s}, \hat{t}, \hat{u}, x_{a}, x_{b}\right) \otimes D_{C / c}\left(z, Q^{2}\right),
\end{aligned}
$$

where $f_{a / A}\left(x_{a}, Q^{2}\right)$ denotes the PDF for parton $a$ inside hadron $A$ carrying a fraction $x_{a}$ of the parent hadron light-cone momentum; $D_{C / c}\left(z, Q^{2}\right)$ is the FF for parton $c$ fragmenting into hadron $C$ with a fraction $z$ of the parton light-cone momentum; $\mathrm{d} \hat{\sigma}^{a b \rightarrow c d}$ denotes the partonic cross section for the elementary scattering $a b \rightarrow c d ; \hat{s}, \hat{t}, \hat{u}$ are the Mandelstam variables for the partonic process.

In order to include $\boldsymbol{k}_{\perp}$ effects we have to generalize standard collinear $\mathrm{PDF}$ and FF to $\boldsymbol{k}_{\perp}$-dependent functions; for instance $f_{a / A}\left(x_{a}\right)$ is generalized to $\hat{f}_{a / A}\left(x_{a}, \boldsymbol{k}_{\perp a}\right)$, where $\boldsymbol{k}_{\perp a}$ is the parton momentum perpendicular to the hadron momentum, with the condition:

$$
f_{a / A}\left(x_{a}\right)=\int \mathrm{d}^{2} \boldsymbol{k}_{\perp a} \hat{f}_{a / A}\left(x_{a}, \boldsymbol{k}_{\perp a}\right) .
$$


Analogously, the fragmentation function $D_{C / c}(z)$ is generalized to $\hat{D}_{C / c}\left(z, \boldsymbol{k}_{\perp C}\right)$, where $\boldsymbol{k}_{\perp C}$ is the transverse momentum of the observed hadron $C$ with respect to the fragmenting parton $c$ momentum.

The next step is to extend the pQCD expression for the cross section, Eq. (3), including $\boldsymbol{k}_{\perp}$ effects:

$$
\begin{aligned}
\mathrm{d} \sigma^{A B \rightarrow C X}= & \sum_{a, b, c, d} \hat{f}_{a / A}\left(x_{a}, \boldsymbol{k}_{\perp a} ; Q^{2}\right) \otimes \hat{f}_{b / B}\left(x_{b}, \boldsymbol{k}_{\perp b} ; Q^{2}\right) \\
& \otimes \mathrm{d} \hat{\sigma}^{a b \rightarrow c d}\left(\hat{s}, \hat{t}, \hat{u}, x_{a}, x_{b}\right) \otimes \hat{D}_{C / c}\left(z, \boldsymbol{k}_{\perp C} ; Q^{2}\right),
\end{aligned}
$$

where now $\hat{s}, \hat{t}$ and $\hat{u}$ depend on the full $\boldsymbol{k}_{\perp}$ kinematics. ${ }^{1}$

A factorization theorem with the inclusion of transverse momenta has not been formally proved in general, ${ }^{2}$ but only for the Drell-Yan process, for two-particle inclusive production in $e^{+} e^{-}$annihilation $^{3}$ and, recently, for SIDIS processes in particular kinematical regions. ${ }^{4}$

In order to study spin asymmetries we have to include explicit spin dependences into Eq. (5). This can be done by introducing in the factorization scheme the parton helicity density matrices describing the parton spin states. In this way, starting from Eq. (5) we can write the polarized cross section as

$$
\begin{gathered}
\mathrm{d} \sigma^{\left(A, S_{A}\right)+\left(B, S_{B}\right) \rightarrow C+X}=\sum_{a, b, c, d,\{\lambda\}} \rho_{\lambda_{a}, \lambda_{a}^{\prime}}^{a / A, S_{A}} \hat{f}_{a / A, S_{A}}\left(x_{a}, \boldsymbol{k}_{\perp a}\right) \otimes \\
\rho_{\lambda_{b}, \lambda_{b}^{\prime}}^{b / B, S_{B}} \hat{f}_{b / B, S_{B}}\left(x_{b}, \boldsymbol{k}_{\perp b}\right) \otimes \hat{M}_{\lambda_{c}, \lambda_{d} ; \lambda_{a}, \lambda_{b}} \hat{M}_{\lambda_{c}^{\prime}, \lambda_{d} ; \lambda_{a}^{\prime}, \lambda_{b}^{\prime}}^{*} \otimes \hat{D}_{\lambda_{c}, \lambda_{c}^{\prime}}^{\lambda_{C}, \lambda_{C}}\left(z, \boldsymbol{k}_{\perp C}\right)(6)
\end{gathered}
$$

where $\{\lambda\}$ is a shorthand for the sum over all helicity indices involved. In Eq. (6), $\rho_{\lambda_{a}, \lambda_{a}^{\prime}}^{a / A, S_{A}}$ is the helicity density matrix of parton $a$ inside hadron $A$ with generic polarization $S_{A}$. Similarly for parton $b$ inside hadron $B$. The $\hat{M}_{\lambda_{c}, \lambda_{d} ; \lambda_{a}, \lambda_{b}}$ 's are the helicity amplitudes for the elementary process $a b \rightarrow c d$, normalized so that the unpolarized cross section, for a collinear collision, is given by

$$
\frac{d \hat{\sigma}^{a b \rightarrow c d}}{d \hat{t}}=\frac{1}{16 \pi \hat{s}^{2}} \frac{1}{4} \sum_{\lambda_{a}, \lambda_{b}, \lambda_{c}, \lambda_{d}}\left|\hat{M}_{\lambda_{c}, \lambda_{d} ; \lambda_{a}, \lambda_{b}}\right|^{2} .
$$

The $\hat{M}_{\lambda_{c}, \lambda_{d} ; \lambda_{a}, \lambda_{b}}$ 's in Eq. (6) are defined in the hadronic c.m. frame; they are related to the usual helicity amplitudes, defined in the "canonical' partonic c.m. frame, $\hat{M}_{\lambda_{c}, \lambda_{d} ; \lambda_{a}, \lambda_{b}}^{0}$, in a non trivial way by proper phases coming from the rotations and the boost connecting the two reference frames. These azimuthal phases are crucial in determining, when integrating over partonic phase space, the size of each allowed contribution to Eq. (6). ${ }^{5,6,7}$ 
Finally, $\hat{D}_{\lambda_{c}, \lambda_{c}^{\prime}}^{\lambda_{C}, \lambda_{C}^{\prime}}\left(z, \boldsymbol{k}_{\perp C}\right)$ is the product of fragmentation amplitudes for the $c \rightarrow C+X$ process

$$
\hat{D}_{\lambda_{c}, \lambda_{c}^{\prime}}^{\lambda_{C}, \lambda_{C}^{\prime}}=\mathcal{F}_{X, \lambda_{X}} \hat{\mathcal{D}}_{\lambda_{X}, \lambda_{C} ; \lambda_{c}} \hat{\mathcal{D}}_{\lambda_{X}, \lambda_{C}^{\prime} ; \lambda_{c}^{\prime}}^{*}
$$

where $\oint_{X, \lambda_{X}}$ stands for a spin sum and phase space integration over all undetected particles, considered as a system $X$. The usual unpolarized fragmentation function $D_{C / c}(z)$, i.e. the number density of hadrons $C$ resulting from the fragmentation of an unpolarized parton $c$ and carrying a light-cone momentum fraction $z$, is given by

$$
D_{C / c}(z)=\frac{1}{2} \sum_{\lambda_{c}, \lambda_{C}} \int d^{2} \boldsymbol{k}_{\perp C} \hat{D}_{\lambda_{c}, \lambda_{c}}^{\lambda_{C}, \lambda_{C}}\left(z, \boldsymbol{k}_{\perp C}\right) .
$$

One can also give a more explicit connection between hadron and parton polarizations. Interpreting the partonic distribution, at $\mathrm{LO}$, as the inclusive cross section for the process $A \rightarrow a+X$ we have:

$$
\begin{aligned}
\rho_{\lambda_{a}, \lambda_{a}^{\prime}}^{a / A, S_{A}} \hat{f}_{a / A, S_{A}}\left(x_{a}, \boldsymbol{k}_{\perp a}\right) & =\sum_{\lambda_{A}, \lambda_{A}^{\prime}} \rho_{\lambda_{A}, \lambda_{A}^{\prime}}^{A, S_{A}} \mathcal{F}_{X_{A}, \lambda_{X_{A}}} \hat{\mathcal{F}}_{\lambda_{a}, \lambda_{X_{A}} ; \lambda_{A}} \hat{\mathcal{F}}_{\lambda_{a}^{\prime}, \lambda_{X_{A}} ; \lambda_{A}^{\prime}}^{*} \\
& =\sum_{\lambda_{A}, \lambda_{A}^{\prime}} \rho_{\lambda_{A}, \lambda_{A}^{\prime}}^{A, S_{A}} \hat{F}_{\lambda_{A}, \lambda_{A}^{\prime}}^{\lambda_{a}, \lambda_{a}^{\prime}}
\end{aligned}
$$

where $\rho_{\lambda_{A}, \lambda_{A}^{\prime}}^{A, S_{A}}$ is the hadron helicity density matrix,

$$
\hat{F}_{\lambda_{A}, \lambda_{A}^{\prime}}^{\lambda_{a}, \lambda_{a}^{\prime}} \equiv \mathcal{F}_{X_{A}, \lambda_{X_{A}}} \hat{\mathcal{F}}_{\lambda_{a}, \lambda_{X_{A}} ; \lambda_{A}} \hat{\mathcal{F}}_{\lambda_{a}^{\prime}, \lambda_{X_{A}} ; \lambda_{A}^{\prime}},
$$

and the $\hat{\mathcal{F}}$ 's are the helicity distribution amplitudes for the $A \rightarrow a+X$ process. Inserting Eq. (10) into Eq. (6) we obtain our master formula for a collision between polarized hadrons:

$$
\begin{aligned}
\mathrm{d} \sigma^{\left(A, S_{A}\right)+\left(B, S_{B}\right) \rightarrow C+X}= & \sum_{a, b, c, d,\{\lambda\}} \rho_{\lambda_{A}, \lambda_{A}^{\prime}}^{A, S_{A}} \hat{F}_{\lambda_{A}, \lambda_{A}^{\prime}}^{\lambda_{a}, \lambda_{a}^{\prime}} \otimes \rho_{\lambda_{B}, \lambda_{B}^{\prime}}^{B, S_{B}} \hat{F}_{\lambda_{B}, \lambda_{B}^{\prime}}^{\lambda_{b}, \lambda_{b}^{\prime}} \\
& \otimes \hat{M}_{\lambda_{c}, \lambda_{d} ; \lambda_{a}, \lambda_{b}} \hat{M}_{\lambda_{c}^{\prime}, \lambda_{d} ; \lambda_{a}^{\prime}, \lambda_{b}^{\prime}}^{*} \otimes \hat{D}_{\lambda_{c}, \lambda_{c}^{\prime}}^{\lambda_{C}, \lambda_{C}}
\end{aligned}
$$

This expression contains all allowed combinations of spin and $\boldsymbol{k}_{\perp}$-dependent distribution and fragmentation functions: at LO and leading twist these functions have a simple partonic interpretation and are related to the spin and $\boldsymbol{k}_{\perp}$-dependent functions discussed in other papers. ${ }^{8,9}$ As an example, 
the relation between the definitions of the Sivers, the Boer-Mulders, and the Collins functions in two widely adopted notations is the following: ${ }^{10}$

$$
\begin{aligned}
f_{q / h^{\uparrow}}-f_{q / h \downarrow} & \equiv \Delta^{N} f_{q / h^{\uparrow}}=4 \operatorname{Im}\left(\hat{F}_{+,-}^{+,+}\right) \propto f_{1 T}^{\perp} \\
f_{q^{\uparrow} / h}-f_{q^{\downarrow} / h} & \equiv \Delta^{N} f_{q^{\uparrow} / h}=2 \operatorname{Im}\left(\hat{F}_{+,-,}^{+,-}\right) \propto h_{1}^{\perp} \\
D_{h / q^{\uparrow}}-D_{h / q^{\downarrow}} & \equiv \Delta^{N} D_{h / q^{\uparrow}}=2 \operatorname{Im}\left(D_{+-}^{h}\right) \propto H_{1}^{\perp} .
\end{aligned}
$$

In Eq. (13) $\Delta^{N} f_{q / h^{\uparrow}}$ gives the probability to find an unpolarized parton inside a transversely polarized hadron. $\Delta^{N} f_{q^{\uparrow} / h}$ in Eq. (14), is the probability to find a transversely polarized quark inside an unpolarized hadron. Finally, $\Delta^{N} D_{h / q^{\uparrow}}$ is the probability for a transversely polarized quark to fragment into an unpolarized hadron.

\section{1. $A_{N}\left(A^{\uparrow} B \rightarrow \pi+X\right)$}

As an application of this formalism let us consider the process $A^{\uparrow} B \rightarrow$ $\pi+X$, where $A$ and $B$ are spin one-half hadrons. In this case the helicity density matrices for $A, B$, take the form:

$$
\rho_{\lambda_{A}, \lambda_{A}^{\prime}}^{A, \uparrow / \downarrow}=\frac{1}{2}\left(\begin{array}{cc}
1 & \mp i \\
\pm i & 1
\end{array}\right) \quad \rho_{\lambda_{B}, \lambda_{B}^{\prime}}^{B, 0}=\frac{1}{2}\left(\begin{array}{ll}
1 & 0 \\
0 & 1
\end{array}\right) .
$$

By performing explicitly the sum over hadron helicity indices in Eq. (12) and using Eq. (16) we obtain:

$$
\begin{array}{r}
\mathrm{d} \sigma^{A^{\uparrow / \downarrow}+B \rightarrow \pi+X}=\sum_{a, b, c, d,\{\lambda\}}\left[\hat{F}_{+,+}^{\lambda_{a}, \lambda_{a}^{\prime}}+\hat{F}_{-,-}^{\lambda_{a}, \lambda_{a}^{\prime}} \mp i\left(\hat{F}_{+,-}^{\lambda_{a}, \lambda_{a}^{\prime}}-\hat{F}_{-,+}^{\lambda_{a}, \lambda_{a}^{\prime}}\right)\right] \\
\otimes\left[\hat{F}_{+,+}^{\lambda_{b}, \lambda_{b}^{\prime}}+\hat{F}_{-,-}^{\lambda_{b}, \lambda_{b}^{\prime}}\right] \otimes \hat{M}_{\lambda_{c} \lambda_{d} ; \lambda_{a} \lambda_{b}} \hat{M}_{\lambda_{c}^{\prime} \lambda_{d} ; \lambda_{a}^{\prime} \lambda_{b}^{\prime}}^{*} \otimes \hat{D}_{\lambda_{c}, \lambda_{c}^{\prime}}^{\pi},
\end{array}
$$

where $\{\lambda\}$ stands for a sum over partonic helicity indices.

In Eq. (17) there are terms that change their sign when changing the sign of the corresponding hadron polarization. These terms survive in the numerator of the asymmetry and involve, depending also on the partonic subprocess considered, different combinations of the Sivers, Boer-Mulders and Collins mechanisms.

As an explicit example let us now consider a particular partonic subprocess: $q q \rightarrow q q$. Then, using known symmetry properties of the helicity distribution functions ${ }^{5}$ we can write:

$$
\begin{aligned}
& \hat{F}_{\lambda_{A}, \lambda_{A}^{\prime}}^{\lambda_{a}, \lambda_{a}^{\prime}}\left(x_{a}, \boldsymbol{k}_{\perp a}\right)=F_{\lambda_{A}, \lambda_{A}^{\prime}}^{\lambda_{a}, \lambda_{a}^{\prime}}\left(x_{a}, k_{\perp a}\right) \exp \left[i\left(\lambda_{A}-\lambda_{A}^{\prime}\right) \phi_{a}\right], \\
& F_{-\lambda_{A},-\lambda_{A}^{\prime}}^{-\lambda_{a},-\lambda_{a}^{\prime}}=(-1)^{\left(\lambda_{A}-\lambda_{a}\right)+\left(\lambda_{A}^{\prime}-\lambda_{a}^{\prime}\right)} F_{\lambda_{A}, \lambda_{A}^{\prime}}^{\lambda_{a}, \lambda_{a}^{\prime}},
\end{aligned}
$$


where $\phi_{i}(i=a, b, c, d)$ are the azimuthal angles of parton $i$ threemomentum in the $A B$ c.m. frame. Analogously for fragmentation functions we have:

$$
\hat{D}_{\lambda_{c}, \lambda_{c}^{\prime}}^{\pi}=D_{\lambda_{c}, \lambda_{c}^{\prime}}^{\pi} \exp \left[i\left(\lambda_{c}-\lambda_{c}^{\prime}\right) \phi_{\pi}^{H}\right]
$$

where $\phi_{\pi}^{H}$ is the azimuthal angle of the pion three-momentum as seen in the parton $c$ helicity frame. ${ }^{5}$

As mentioned above, the helicity amplitudes $\hat{M}_{\lambda_{c} \lambda_{d} ; \lambda_{a} \lambda_{b}}$ in Eq. (17) are defined in the hadronic c.m. frame; we can relate these amplitudes to those given in the canonical partonic c.m. frame, $\hat{M}_{\lambda_{c} \lambda_{d} ; \lambda_{a} \lambda_{b}}^{0}$, where $Z$ is the direction of the colliding partons and the $X Z$-plane coincides with the scattering plane. In this frame the $\hat{M}_{\lambda_{c} \lambda_{d} ; \lambda_{a} \lambda_{b}}^{0}$ 's exhibit explicit symmetry properties.

To reach the canonical partonic c.m. frame, from the hadronic c.m. frame, we have first to perform a boost along the $\boldsymbol{p}_{a}+\boldsymbol{p}_{b}$ direction, so that the boosted three-vector $\boldsymbol{p}_{a}^{\prime}+\boldsymbol{p}_{b}^{\prime}$ is vanishing. In this frame $\left(S^{\prime}\right)$ partons $a$ and $b$ are in the "head-on" configuration, but not aligned along the $Z$-axis direction. We then perform a rotation to align the colliding initial partons with the $Z$-axis. We call this new frame $S^{\prime \prime}$. In this frame parton $c$ three-momentum does not lie in the $X Z$-plane but has a transverse component, with an azimuthal angle $\phi_{c}^{\prime \prime}$. A final rotation around $Z$ by $\phi_{c}^{\prime \prime}$ leads to the canonical configuration. The relationship between $\hat{M}_{\lambda_{c} \lambda_{d} ; \lambda_{a} \lambda_{b}}$ and $\hat{M}_{\lambda_{c} \lambda_{d} ; \lambda_{a} \lambda_{b}}^{0}$ is then given by: ${ }^{5}$

$$
\begin{aligned}
& \hat{M}_{\lambda_{c}, \lambda_{d} ; \lambda_{a}, \lambda_{b}}=\hat{M}_{\lambda_{c}, \lambda_{d} ; \lambda_{a}, \lambda_{b}}^{0} \\
& \times e^{-i\left[\lambda_{a}\left(\xi_{a}+\tilde{\xi}_{a}\right)+\lambda_{b}\left(\xi_{b}+\tilde{\xi}_{b}\right)-\lambda_{c}\left(\xi_{c}+\tilde{\xi}_{c}\right)-\lambda_{d}\left(\xi_{d}+\tilde{\xi}_{d}\right)\right]} e^{i\left(\lambda_{a}-\lambda_{b}\right) \phi_{c}^{\prime \prime}} \\
& \quad=\hat{M}_{\lambda_{c}, \lambda_{d} ; \lambda_{a}, \lambda_{b}}^{0} \\
& \times e^{-i\left(\lambda_{a} \xi_{a}+\lambda_{b} \xi_{b}-\lambda_{c} \xi_{c}-\lambda_{d} \xi_{d}\right)} e^{-i\left[\left(\lambda_{a}-\lambda_{b}\right) \tilde{\xi}_{a}-\left(\lambda_{c}-\lambda_{d}\right) \tilde{\xi}_{c}\right]} e^{i\left(\lambda_{a}-\lambda_{b}\right) \phi_{c}^{\prime \prime}},
\end{aligned}
$$

where $\xi_{i}, \tilde{\xi}_{i}(i=a, b, c, d)$ are phases due to the behaviour of helicity states under the Lorentz transformations connecting the hadronic and the canonical partonic c.m. frame..$^{5,11}$

Using Eqs. (18), (19), (20), (22) into Eq. (17) we obtain the contribution 
to the numerator of $A_{N}$ coming from the $q q \rightarrow q q$ partonic subprocess:

$$
\begin{aligned}
& \mathrm{d} \sigma_{q q \rightarrow q q}^{A^{\uparrow} B \rightarrow \pi X}-\mathrm{d} \sigma_{q q \rightarrow q q}^{A^{\downarrow} B \rightarrow \pi X} \propto \\
& 2 \operatorname{Im}\left[\hat{F}_{+-}^{++}\left(x_{a}, k_{\perp a}\right)\right] \cos \phi_{a} \otimes \hat{f}_{b / B}\left(x_{b}, k_{\perp b}\right) \\
& \quad\left(\left|\hat{M}_{++;++}^{0}\right|^{2}+\left|\hat{M}_{-+;-+}^{0}\right|^{2}+\left|\hat{M}_{-+;+-}^{0}\right|^{2}\right) \otimes \hat{D}_{++}^{\pi} \\
& -\left\{\hat{F}_{+-}^{+-}\left(x_{a}, k_{\perp a}\right) \cos \left(2 \phi_{c}^{\prime \prime}-\xi_{a}-\tilde{\xi}_{a}+\xi_{b}+\tilde{\xi}_{b}+\phi_{a}\right)\right\} \\
& \left.-\hat{F}_{+-}^{-+}\left(x_{a}, k_{\perp a}\right) \cos \left(2 \phi_{c}^{\prime \prime}-\xi_{a}-\tilde{\xi}_{a}+\xi_{b}+\tilde{\xi}_{b}-\phi_{a}\right)\right\} \\
& \quad \otimes \operatorname{Im}\left[\hat{F}_{++}^{+-}\left(x_{b}, k_{\perp b}\right)\right] \otimes \hat{M}_{-+;+-}^{0} \hat{M}_{-+;-+}^{0} \otimes \hat{D}_{++}^{\pi} \\
& +\left\{\hat{F}_{+-}^{+-}\left(x_{a}, k_{\perp a}\right) \cos \left(\phi_{c}^{\prime \prime}+\phi_{\pi}^{H}-\xi_{a}-\tilde{\xi}_{a}+\xi_{c}+\tilde{\xi}_{c}+\phi_{a}\right)\right. \\
& \left.-\hat{F}_{+-}^{-+}\left(x_{a}, k_{\perp a}\right) \cos \left(\phi_{c}^{\prime \prime}+\phi_{\pi}^{H}-\xi_{a}-\tilde{\xi}_{a}+\xi_{c}+\tilde{\xi}_{c}-\phi_{a}\right)\right\} \\
& \quad \otimes \hat{f}_{b / B}\left(x_{b}, k_{\perp b}\right) \otimes \hat{M}_{++;++}^{0} \hat{M}_{-+;-+}^{0} \otimes 2 \operatorname{Im}\left(\hat{D}_{+-}^{\pi}\right) \\
& -8 \operatorname{Im}\left[\hat{F}_{+-}^{++}\left(x_{a}, k_{\perp a}\right)\right] \cos \phi_{a} \otimes \operatorname{Im}\left[\hat{F}_{++}^{+-}\left(x_{b}, k_{\perp b}\right)\right] \\
& \quad \times \cos \left(\phi_{c}^{\prime \prime}-\phi_{\pi}^{H}+\xi_{b}+\tilde{\xi}_{b}-\xi_{c}-\tilde{\xi}_{c}\right) \otimes \hat{M}_{++;++}^{0} \hat{M}_{-+;+-}^{0} \otimes \operatorname{Im}\left(\hat{D}_{+-}^{\pi}\right) .
\end{aligned}
$$

With the help of Eqs. (13), (14) and (15) one can identify in this expression the Sivers, Boer-Mulders and Collins mechanisms. The terms $\hat{F}_{+-}^{+-}$ and $\hat{F}_{+-}^{-+}$are in turn related to the distribution of transversely polarized quarks inside a transversely polarized hadron, the well-known transversity function.

\section{Final hadron polarization in hadronic collisions}

Within the same formalism we are able to calculate polarized cross sections for processes in which the final, spin one-half hadron (e.g. a $\Lambda$ hyperon), is polarized. Our master formula, Eq. (12), is modified by the introduction of the helicity density matrix $\rho_{\lambda_{\Lambda}, \lambda_{\Lambda}^{\prime}}^{\Lambda}$ of the observed hadron, describing its polarization state. This way, Eq. (12) becomes:

$$
\begin{aligned}
& \rho_{\lambda_{\Lambda}, \lambda_{\Lambda}^{\prime}}^{\Lambda} \mathrm{d} \sigma^{\left(A, S_{A}\right)\left(B, S_{B}\right) \rightarrow \Lambda X} \propto \sum_{\lambda_{X}, X} \sum_{\lambda_{c}, \lambda_{c}^{\prime}} \hat{\mathcal{D}}_{\lambda_{\Lambda}, \lambda_{X}, \lambda_{c}} \hat{\mathcal{D}}_{\lambda_{\Lambda}^{\prime}, \lambda_{X}, \lambda_{c}^{\prime}}^{*} \rho_{\lambda_{c}, \lambda_{c}^{\prime}}^{\Lambda / c} \\
& =\sum_{a, b, c, d,\{\lambda\}} \rho_{\lambda_{A}, \lambda_{A}^{\prime}}^{A, S_{A}} \hat{F}_{\lambda_{A}, \lambda_{A}^{\prime}}^{\lambda_{a}, \lambda_{a}^{\prime}} \otimes \rho_{\lambda_{B}, \lambda_{B}^{\prime}}^{B, S_{B}} \hat{F}_{\lambda_{B}, \lambda_{B}^{\prime}}^{\lambda_{1}, \lambda_{b}^{\prime}} \otimes \hat{M}_{\lambda_{c} \lambda_{d} ; \lambda_{a} \lambda_{b}} \hat{M}_{\lambda_{c}^{\prime} \lambda_{d} ; \lambda_{a}^{\prime} \lambda_{b}^{\prime}}^{*} \otimes \hat{D}_{\lambda_{c}, \lambda_{c}^{\prime}}^{\lambda_{\Lambda}, \lambda_{\Lambda}^{\prime}} .
\end{aligned}
$$

By choosing $S_{A}$ and $S_{B}$, and performing the sum over partonic helicity indices (as discussed above), Eq. (23) allows one to compute all polarization 
states (longitudinal and transverse) for a final spin- $1 / 2$ hadron produced in (un)polarized hadron-hadron collisions. Notice also the appearance in the fragmentation sector of new terms with respect to the pion case, depending on the final hadron helicities.

\section{Conclusions}

Spin effects in inclusive high-energy hadronic reactions play an important role in our understanding of strong interactions.

We have presented a general approach to describe, within pQCD factorization schemes and using the helicity formalism, polarized inclusive particle production in high-energy hadronic collisions. By taking into account intrinsic motion of partons in the distribution and fragmentation functions and in the partonic process, this approach allows one to give explicit expressions for single and double spin asymmetries. This requires the introduction of a new class of spin and $\boldsymbol{k}_{\perp}$-dependent functions. The combined study of single and double spin asymmetries for different particles and in different kinematical situations may help in gathering information on these basically unknown functions. As an example, we have briefly discussed two interesting applications of this approach, namely $A_{N}\left(A^{\uparrow} B \rightarrow \pi+X\right)$ and final hadron polarization in (un)polarized hadronic collisions.

\section{References}

1. U. D'Alesio and F. Murgia, Phys. Rev. D70 (2004) 074009

2. J.C. Collins, Nucl. Phys. B396 (1993) 161

3. J.C. Collins, D.E. Soper and G. Sterman, Nucl. Phys. B250 (1985) 199; J.C. Collins and D.E. Soper, Nucl. Phys. B193 (1981) 381

4. X. Ji, J.-P. Ma and F. Yuan, e-Print Archive: hep-ph/0404183; Phys. Lett. B597 (2004) 299

5. M. Anselmino, M. Boglione, U. D'Alesio, E. Leader and F. Murgia e-Print Archive: hep-ph/0408356 (Phys. Rev. D, in press)

6. M. Anselmino, M. Boglione, U. D'Alesio, E. Leader, S. Melis and F. Murgia in preparation

7. M. Anselmino, M. Boglione, U. D'Alesio, E. Leader and F. Murgia, Phys. Rev. D70 (2004) 074025

8. D. Boer, P. Mulders and F. Pijlman, Nucl. Phys. B667 (2003) 201

9. V. Barone, A. Drago and P. Ratcliffe, Phys. Rep. 359 (2002) 1

10. A. Bacchetta, U. D'Alesio, M. Diehl, C. Andy Miller, e-Print Archive: hepph/0410050 (Phys. Rev. D, in press)

11. For a pedagogical introduction to all the basics of helicity formalism, see, e.g., E. Leader, Spin in Particle Physics, Cambridge University Press, 2001 